\title{
Gas-Induced Segregation in Pt-Rh Alloy Nanoparticles Observed by In Situ Bragg Coherent Diffraction Imaging
}

\author{
Tomoya Kawaguchi $\odot,{ }^{1,6}$ Thomas F. Keller $\odot,{ }^{2,3}$ Henning Runge $\odot,{ }^{2,3}$ Luca Gelisio $\odot,{ }^{2}$ Christoph Seitz, ${ }^{2}$ Young

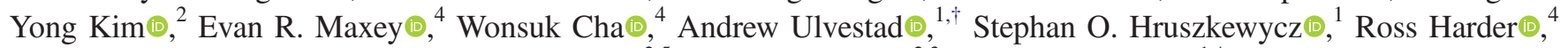 \\ Ivan A. Vartanyants $\odot,{ }^{2,5}$ Andreas Stierle $\odot,{ }^{2,3}$ and Hoydoo You $\odot^{1, *}$ \\ ${ }^{1}$ Materials Science Division, Argonne National Laboratory, Argonne, Illinois 60439, USA \\ ${ }^{2}$ Deutsches Elektronen-Synchrotron DESY, D-22603 Hamburg, Germany \\ ${ }^{3}$ Physics Department, Universität Hamburg, D-20355 Hamburg, Germany \\ ${ }^{4}$ Advanced Photon Source, Argonne National Laboratory, Argonne, Illinois 60439, USA \\ ${ }^{5}$ National Research Nuclear University MEPhI, 115409 Moscow, Russia \\ ${ }^{6}$ Institute for Materials Research, Tohoku University, Sendai, 9808577, Japan
}

(Received 16 May 2019; published 13 December 2019)

\begin{abstract}
Bimetallic catalysts can undergo segregation or redistribution of the metals driven by oxidizing and reducing environments. Bragg coherent diffraction imaging (BCDI) was used to relate displacement fields to compositional distributions in crystalline Pt-Rh alloy nanoparticles. Three-dimensional images of internal composition showed that the radial distribution of compositions reverses partially between the surface shell and the core when gas flow changes between $\mathrm{O}_{2}$ and $\mathrm{H}_{2}$. Our observation suggests that the elemental segregation of nanoparticle catalysts should be highly active during heterogeneous catalysis and can be a controlling factor in synthesis of electrocatalysts. In addition, our study exemplifies applications of BCDI for in situ 3D imaging of internal equilibrium compositions in other bimetallic alloy nanoparticles.
\end{abstract}

DOI: 10.1103/PhysRevLett.123.246001

Transition metals and their metal alloys have been extensively studied as materials for heterogeneous catalysis and electrocatalysis. In particular, metals in the platinum group and their alloys are among the most important catalysts for organic and electrochemical reactions [1,2]. In industrial applications, they are typically used as nanoparticles to increase efficiency and selectivity. For this reason, the size-shape-activity relationships have been a focus of extensive studies and the importance of surface reactions has been recognized $[3,4]$. In some studies, it was further suggested that the shapes and sizes of the nanoparticles can change during reactions. For example, the surface compositions of bimetallic nanoparticles were reported to change depending on oxidizing or reducing environments $[5,6]$. Since the changes occur during reactions, studies must be carried out in situ in detail on an individual nanoparticle to fully understand the internal dynamics of elemental distributions.

A Pt-Rh alloy nanoparticle is an excellent system to study the effect of surface reactions on the compositional redistributions. The unit cell structures of both $\mathrm{Pt}$ and $\mathrm{Rh}$ are face-centered cubic (fcc) with $3 \%$ differences in lattice constants $\left(a_{\mathrm{Pt}}=3.9242 \AA, a_{\mathrm{Rh}}=3.8034 \AA\right)$. In bulk, they form solid solutions with no phase separation [7] and negligible compositional ordering [8]. Since there are no significant internal driving forces for compositional redistribution, external driving forces by surface reactions can have a significant effect. If the effect of the surface reactions can be detected in a particle with a diameter of $\sim 100 \mathrm{~nm}$, which is roughly the size needed in this study for a suitable signal-to-noise ratio, we expect that the effect must be significant and relevant for real catalyst nanoparticles with higher surface-to-volume ratios.

Bragg coherent diffraction imaging (BCDI) is increasingly popular for imaging single-crystal nanoparticles under in situ conditions [9-11]. The 3D real-space images reconstructed using BCDI are composed of voxels of complex numbers. The amplitude of the voxel is in principle proportional to the electron density of the crystalline order [12,13], while the phase is proportional to the displacement of the crystallographic planes [14-16]. However, the degree of crystalline order within the particle is generally unknown and the amplitude alone can be unreliable in measuring electron densities. On the other hand, the phase of a BCDI image is sensitive to lattice displacements. The phase can also in principle be affected by the refraction effect [17] and the dynamical scattering effect $[18,19]$. However, because the nanoparticle size in our measurements is small compared to the extinction length or the Pendellösung length, the dynamical effects are small and the phase is dominantly proportional to the lattice displacement $[18,19]$. Therefore, use of the phase image guided by the amplitude image can provide the reliable composition distribution of a binary alloy nanoparticle by relating the lattice spacings to the local compositions.

The lattice spacing can be obtained from the atomic displacement field $u_{111}(\boldsymbol{r})$ of the phase image reconstructed 
in BCDI. First, the lattice spacing deviation from the average is obtained from the derivative of the longitudinal displacement, $\partial_{111} u_{111}(\boldsymbol{r}) / \partial r_{111}[16,20]$. In the case of electrochemical dealloying [20], the deviation was used to map the mechanical strain at room temperature induced by the dealloying process. In the case of an alloy nanoparticle at high temperatures, however, the alloy elements can diffuse freely and the mechanical strain can be replaced by the compositional strain, i.e., the strain induced by the compositional heterogeneity [21-26]. Then, the local composition can be determined from the compositional strain via Vegard's law assuming that the changes in local composition isotopically expand or contract the cubic unit cell [27]. Thus, we will present our results as 3D images of local $\mathrm{Rh}$ compositions converted from the phase [21] and 3D images of normalized electron densities.

Figure 1 shows a scanning electron microscopy (SEM) image of the sample nanoparticle with a nominal $\mathrm{Pt}_{2 / 3}-\mathrm{Rh}_{1 / 3}$ composition [Fig. 1(a)], a slice of the Bragg diffraction data from the particle [Fig. 1(b)], and a 3D shape reconstructed from the data [Fig. 1(c)]. The sample was prepared by depositing a $\mathrm{Rh}$ layer at $630^{\circ} \mathrm{C}$ over $\mathrm{Pt}$ nanoparticles dewetted on $\mathrm{Al}_{2} \mathrm{O}_{3}(0001)$ substrate. Among several particles examined and tested [21], the nanoparticle shown in Fig. 1(a) was selected for detailed studies. Hierarchical fiducial markers were used to identify the same particle with a SEM and with a confocal microscope in the beam line. The 3D shape [Fig. 1(c)] and 3D phase were reconstructed by iterative phase retrieval algorithms standard for BCDI $[28,29]$ in combination with a guided genetic algorithm [30] after applying flat-field correction on the detector and subtracting background scattering. The voxel size of BCDI images was $\sim 6.7 \mathrm{~nm}$
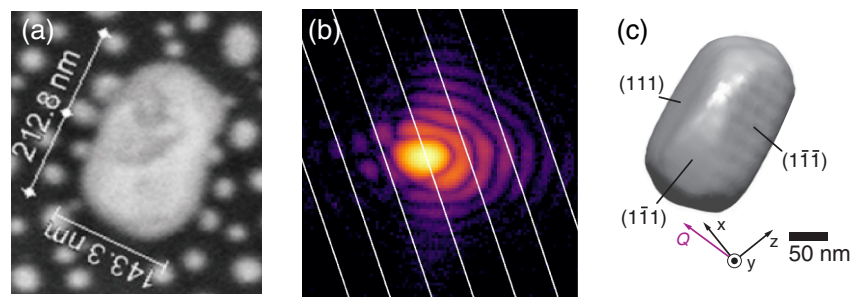

FIG. 1. (a) A SEM image of the $\mathrm{Pt}_{2 / 3}-\mathrm{Rh}_{1 / 3}$ alloy nanoparticle obtained after the BCDI measurements. The smaller nanoparticles in the SEM image are Rh particles. (b) A 2D coherent diffraction pattern of the 111 peak from the particle in a logarithmic intensity scale. The white equi- $2 \theta$ lines indicate the expected Bragg peak maximum for every $10 \%$ change in the mean $\mathrm{Rh}$ composition of the particle. (c) A 3D reconstruction of the same particle using BCDI in a top-down view as is in (a). Isosurface of $45 \%$ of the mean electron density is drawn. $x, y$, and $z$ of the laboratory coordinate system correspond to the radial direction of the synchrotron ring, the vertical direction, and the incident x-ray direction, respectively. The slightly larger size and the smoother shape of (c) compared with (a) are due the BCDI resolution lower than that of SEM. and the resolution was $\sim 13 \mathrm{~nm}$ [21]. The diffraction pattern did not change over the long $\mathrm{x}$-ray exposure indicating that $\mathrm{x}$-ray-induced oxidations were negligible, which is consistent with prior observations on the noble metals [31].

The sample temperature was independently estimated from the $\mathrm{Al}_{2} \mathrm{O}_{3}$ (0006) substrate peak position at each setting temperature based on the thermal expansion known in the literature [32]. Then, the average composition of the sample nanoparticle at that temperature was determined from the Bragg scattering angle of the nanoparticle from the known thermal expansion rates of the Pt-Rh alloys [33,34]. This provided us a means of determining the particle average composition precisely for any given environment and temperature. The initial average composition at $550{ }^{\circ} \mathrm{C}$ determined in this way was close to our target composition of $\mathrm{Pt}_{2 / 3} \mathrm{Rh}_{1 / 3}$ although the composition evolved continuously depending on the environment and temperature during the course of the experiments [21].

The BCDI measurements were carried out under variable gas environments at atmospheric pressures: (i) pure $\mathrm{He}$, (ii) $\mathrm{He}$ with $2.7 \% \mathrm{O}_{2}$, (iii) $\mathrm{He}$ with $5 \% \mathrm{O}_{2}$, and (iv) $\mathrm{He}$ with $3.8 \% \mathrm{H}_{2}$, at two temperatures, $550{ }^{\circ} \mathrm{C}$ and $700{ }^{\circ} \mathrm{C}$. The environments were chosen for (i) neutral, (ii) and (iii) oxidizing, and (iv) reducing conditions, and the two temperatures were chosen for different $\mathrm{Rh}$ diffusion lengths. Rhodium atoms in a Pt matrix are expected to diffuse 6 and $70 \mathrm{~nm}$ at these temperatures, respectively $[35,36]$, over the diffusion time of $10^{3} \mathrm{sec}$, which is close to the approximate wait time at each gas change. The diffusion length at $550^{\circ} \mathrm{C}$ was not sufficient to achieve a full thermal equilibrium over the entire particle but was sufficient for composition redistributions to be seen in BCDI measurements. At the elevated temperature of $700{ }^{\circ} \mathrm{C}$, the particle-average lattice spacing decreased significantly over time [21], indicating the alloy nanoparticles slowly incorporated $\mathrm{Rh}$ atoms from neighboring $\mathrm{Rh}$ nanoislands during the experiment. The $\mathrm{Rh}$ incorporation was not significant at $550^{\circ} \mathrm{C}$, but the lattice spacing responded whenever the gas flows were changed. The measurements at $400{ }^{\circ} \mathrm{C}$ or below showed little effect of gas environments [21], as expected from the extremely short thermal diffusion lengths of $0.15 \mathrm{~nm}$ for $10^{3} \mathrm{sec}$ diffusion.

Figure 2 shows the 3D images of normalized amplitude [Fig. 2(a)] and composition difference [Fig. 2(b)] reconstructed at $550^{\circ} \mathrm{C}$. The $\mathrm{H}_{2}$ exposure significantly decreased the electron density of the particle as indicated by the irregularities in the isosurfaces shown in Fig. 2(a), while the exposures of $\mathrm{He}$ and $\mathrm{O}_{2}$ have almost no effect on the particle shape. While the density irregularity suggests an increased $\mathrm{Rh}$ composition, it can also indicate a significant reduction in crystalline order $[12,13]$ as discussed earlier. Hence, the composition images obtained from the phase [21] are shown in Fig. 2(b) as the cross-sectional views of the Rh-composition variations. In an oxidizing atmosphere $\left(\mathrm{O}_{2}\right)$, only subtle growth of the Pt-rich region (green region) 
(a)
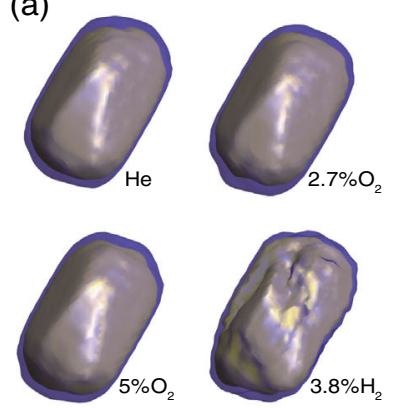

(b)

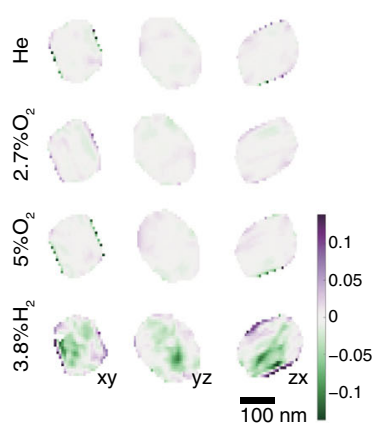

(c)
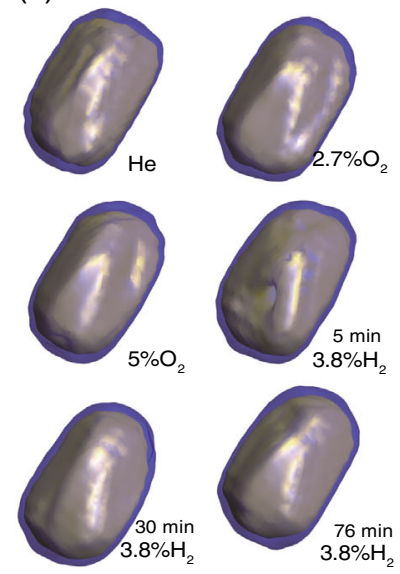

(d)

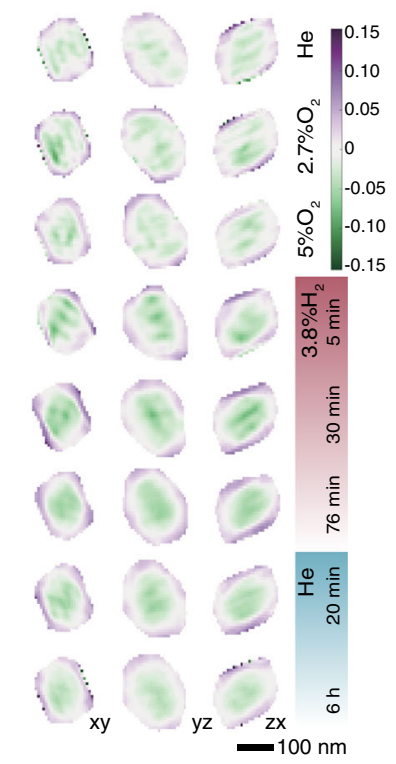

FIG. 2. (a),(c) Electron density normalized by the maximum amplitude and (b),(d) Rh composition difference from the particle average, obtained from compositional strain at various gas atmospheres at $550^{\circ} \mathrm{C}$ (a),(b) and at $700^{\circ} \mathrm{C}$ (c),(d). (a),(c) Translucent isosurfaces at $45 \%$ (blue envelope) and $85 \%$ (yellow core) of the mean electron density are shown. See the movie files in Supplemental Material for 3D renderings with the $45 \%$ and $85 \%$ isosurfaces [21]. (b),(d) The color bar indicates the $\mathrm{Rh}$ composition difference $\left(\Delta x_{\mathrm{Rh}}=x_{\mathrm{Rh}}-\bar{x}_{\mathrm{Rh}}\right)$ [21], where $\bar{x}_{\mathrm{Rh}} \simeq$ 0.33 and $x_{\mathrm{Rh}}$ ranges from $\sim 0.18$ (green) to $\sim 0.48$ (purple) depending on the exact value of the particle average $\bar{x}_{\mathrm{Rh}}$.

was observed near the surface. A reducing atmosphere $\left(\mathrm{H}_{2}\right)$, however, significantly affected the diffraction patterns [21] and the composition accordingly, as Rh-rich regions (purple region) formed and composition variations within the particle became significant.

The particle shape obtained from BCDI remained largely unchanged at $700^{\circ} \mathrm{C}$ and the electron density isosurfaces in Fig. 2(c) appeared in sensitive to gas environments. However, upon a close inspection of the images in Fig. 2(c), some shape change can be seen in the $\mathrm{H}_{2}$ atmosphere at the initial $5 \mathrm{~min}$ exposure of $3.8 \% \mathrm{H}_{2}$. The shape was restored in the images for the 30 and

76 min exposures, presumably as the system reached a steady state or a full thermal equilibrium because of the faster diffusion of $\mathrm{Rh}$ at this temperature. The compositions, shown in Fig. 2(d), indicate a significant $\mathrm{Rh}$ segregation near the surface in all gas environments.

The phase images were further analyzed to obtain the radial distributions of the composition. The radial distributions in Fig. 3 were calculated as a function of the distance from the $45 \%$ isosurface defined in Fig. 2. The radial distance of a voxel was defined as the distance from the nearest voxel on the $45 \%$ isosurface. Then, the radial distance up to $60 \mathrm{~nm}$ measured from the surface was divided to 25 shells and the Rh compositions were averaged at each shell. The number of the voxels was typically $\sim 700$ at the surface compared to $\sim 30$ at the core of the particle [21]. The errors in the radial distribution were estimated from the variance of the averaged values and the number of the voxels. The zero of the Rh composition ( $y$ axis) was set to the compositions averaged for the core of the particle, defined by the radial distance from the surface larger than $40 \mathrm{~nm}$, to show clearly the differences at the surface.

In Fig. 3(a), the radial distribution for the $550{ }^{\circ} \mathrm{C}$ images clearly demonstrates the sensitivity of the surface composition to the gas environments. In the $5 \% \mathrm{O}_{2}$ environment, the Pt-rich (Rh-poor) region appeared at the bins near the surface $(<15 \mathrm{~nm})$ while the composition is relatively flat in $\mathrm{He}$ and in $2.7 \% \mathrm{O}_{2}$. At the same time, the average $\mathrm{Rh}$ composition (inset) decreased slightly under the $2.7 \% \mathrm{O}_{2}$ atmosphere, which continued under $5 \% \mathrm{O}_{2}$. The decrease of the average $\mathrm{Rh}$ composition continues (inset) and $\mathrm{Rh}$ composition depletes further near the surface (diamond). On the other hand, the $\mathrm{H}_{2}$ atmosphere yields a $6 \%$ increase of $\mathrm{Rh}$ composition due to $\mathrm{Rh}$ segregation at the surface as (a)

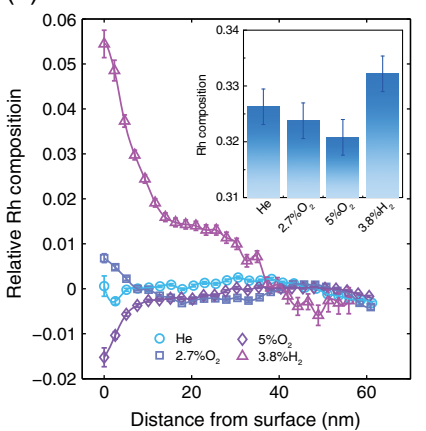

(b)

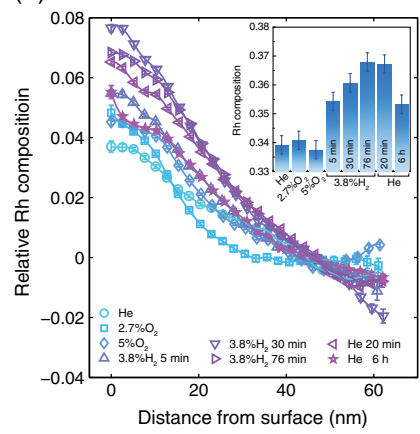

FIG. 3. Radial distributions of the relative $\mathrm{Rh}$ composition converted from compositional strain at $550{ }^{\circ} \mathrm{C}$ (a) and at $700{ }^{\circ} \mathrm{C}$ (b). The zeros of the relative $\mathrm{Rh}$ compositions were set to the mean values of the compositions in the core of $40 \mathrm{~nm}$ or larger from the surface. The displayed times in (b) are the elapsed times after the gas introductions. The insets exhibit the particle average compositions of $\mathrm{Rh}$ determined from the Bragg angles. These values are used to set the zeros in the vertical scale bars in Fig. 2. Error bars in the inset were estimated from the pixel size of the $2 \mathrm{D}$ detector. 
well as a considerable increase of the average Rh composition. The relatively large error bars and the step in the composition around $30 \mathrm{~nm}$ are due to the compositional fluctuation inside the particle, as seen in Fig. 2(b). Averaging the $\mathrm{Rh}$ distribution in the $30 \mathrm{~nm}$ shell is not as smooth as the surface shells because only small number of voxels were averaged while there are a still significant compositional fluctuations.

In Fig. 3(b) at $700^{\circ} \mathrm{C}$, the increased $\mathrm{Rh}$ compositions near the surface are evident regardless of the gas environments, even under $\mathrm{O}_{2}$ flow. However, the trend of the $\mathrm{Rh}$ surface segregation in $\mathrm{H}_{2}$ (upper triangle, down triangle, right triangle) was still consistent with the results at $550{ }^{\circ} \mathrm{C}$. The increased $\mathrm{Rh}$ composition at the surface also led to a steady increase of the overall Rh composition, as shown in the inset. When the gas was switched back to He (left triangle and star), however, the Rh-rich surface region and the overall $\mathrm{Rh}$ composition decreased back slowly with time. In He flow, the composition slope decreased but still remained even after $6 \mathrm{~h}$.

Our results show that the compositional distribution of $\mathrm{Pt}-\mathrm{Rh}$ alloy nanoparticles is dynamic at high temperatures and sensitive to the environment condition. Some rationale to the observed behavior is illustrated in Fig. 4. Since the sample was exposed to air after preparation, the particle surface was initially covered by native oxides of rhodium because rhodium can form crystalline oxides such as hexagonal $\mathrm{Rh}_{2} \mathrm{O}_{3}$ or rutile $\mathrm{RhO}_{2}$ while platinum does not easily form crystalline oxides [37]. The native oxides are expected to be extremely thin, limited to a few atomic layers, as illustrated in Fig. 4(a), since the sample is cooled to room temperature before exposure to air. Note that any diffraction from the oxides, thin or thick, unlikely contributes to the 111 Bragg peak measured here because of its different crystalline structure. Nonetheless, Rh oxide formation is expected and can be a driving force for the observed compositional redistribution in oxidizing environments $[6,38]$.

When the particle is heated in the $\mathrm{O}_{2}$ atmosphere, $\mathrm{Rh}$, being more oxophilic than Pt [39], forms oxides faster and thicker than Pt does. This causes Rh to be dealloyed at the surface of the particle by forming $\mathrm{Rh}$ oxides, which

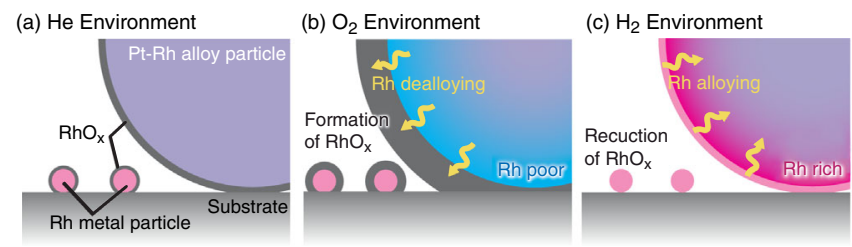

FIG. 4. Schematics of alloying and dealloying processes in $\mathrm{He}$, $\mathrm{O}_{2}$, and $\mathrm{H}_{2}$ environments. (a) In $\mathrm{He}$, the surfaces of the Pt-Rh particle and $\mathrm{Rh}$ nanoparticles all have a thin native oxide layer. (b) In $\mathrm{O}_{2}, \mathrm{Rh}$ oxide layer grows faster because $\mathrm{Rh}$ is more oxophilic than Pt. (c) In $\mathrm{H}_{2}$, the Rh oxide layer reduces, which increases the Rh compositions at the surface. eventually yields the Rh-poor, Pt-rich region on the surface as in Fig. 4(b). In the $\mathrm{H}_{2}$ atmosphere, the reverse reaction is expected. The $\mathrm{Rh}$ oxide is reduced and $\mathrm{Rh}$ atoms are incorporated to the particle. This simple oxidationreduction model does not completely explain the results that the $\mathrm{Rh}$ was significantly enriched in the $\mathrm{H}_{2}$ atmosphere. If the $\mathrm{Rh}$ oxides formed in $\mathrm{O}_{2}$ simply incorporated back to the particle in $\mathrm{H}_{2}$, the $\mathrm{Rh}$ composition on the surface could not have increased as significantly as observed.

The composition increase of Rh at the surface was 0.06 , as shown in Fig. 3(a). The actual surface composition profile can be much narrower and higher because the distribution is a convolution of the actual distribution with the BCDI resolution $(13 \mathrm{~nm})$. Such significant surface segregation can occur only if external $\mathrm{Rh}$ atoms, diffused from neighboring $\mathrm{Rh}$ nanoislands, are incorporated to the surface of the particle. Although we have no direct evidence, it is likely even at $550^{\circ} \mathrm{C}$ that $\mathrm{Rh}$ atoms shuttle back and forth from the neighboring $\mathrm{Rh}$ nanoislands surrounding the $\mathrm{Pt}-\mathrm{Rh}$ particle of this study. The $\mathrm{Rh}$ migration explains naturally the Rh-rich surface. This also increases the overall $\mathrm{Rh}$ composition as illustrated in Fig. 4(c), which could not have taken place without the $\mathrm{Rh}$ migration.

At the relatively high temperature of $700{ }^{\circ} \mathrm{C}$, the situation may be different. The equilibrium oxygen partial pressure of the $\mathrm{Rh}$ oxidation is quite high at $\sim 10^{-2}$ atm [39], which means that $\mathrm{Rh}$ oxides tend to spontaneously reduce to metal in the $\mathrm{He}$ atmosphere. Thus, alloying $\mathrm{Rh}$ by the $\mathrm{Rh}$ migration can continue at this temperature without the $\mathrm{Rh}$ oxide on the surface in $\mathrm{He}$ atmosphere as well as in $\mathrm{H}_{2}$ atmosphere. As discussed earlier, the diffusion length of $\mathrm{Rh}$ at this temperature is $70 \mathrm{~nm}$ at $10^{3} \mathrm{sec}$ that is as large as the average radius of the particle, allowing the entire particle to reach a full thermodynamic equilibrium whenever the gas environment is changed. This explains why the radial distributions appear all similar in the different gases. However, it still shows significant changes of the Rh composition near the surface depending on the gas flow, much like the case at $550^{\circ} \mathrm{C}$. The continuous $\mathrm{Rh}$ migration from the $\mathrm{Rh}$ nanoislands explains the overall slope of the gradual decrease of $\mathrm{Rh}$ from the surface to the core. However, it is also possibly driven thermodynamically toward the truncated surface [3], as experimentally observed in Rh-Pt nanoparticles [40], which requires further theoretical clarification.

In conclusion, the BCDI technique, highly sensitive to nanoscale variations of lattice constants, is shown to be a powerful technique in studying in situ 3D internal composition map and dynamic shell-core compositional rearrangement in a $\mathrm{Pt}_{2 / 3}-\mathrm{Rh}_{1 / 3}$ alloy nanoparticle. At the intermediate temperature of $550^{\circ} \mathrm{C}$, the surface $\mathrm{Rh}$ composition significantly decreases in an oxidizing environment and increases in a reducing environment. At higher temperatures, the Rh composition is significantly higher in the surface regions and gradually decreases toward the core 
of the particle regardless of the gas environments. However, the composition distribution shows gas dependences similar to the results at $550{ }^{\circ} \mathrm{C}$. The redistributions of metals observed in alloy nanoparticles as large as $~ 100 \mathrm{~nm}$ offers an insight into the dynamic nature of the generally much smaller nanoparticle catalysts in practical usages, in particular for gas-phase catalysis and in fuel-cell catalysts operating at elevated temperatures, suggesting that the active compositional segregation, redistribution, and other structural dynamics must be taken into account for rational design of new catalysts and electrocatalysts. This study also opens a possibility of composition imaging studies on other binary alloys in equilibrium under various extreme environmental conditions.

Bragg coherent diffraction imaging x-ray measurements and data analysis was supported by the U.S. Department of Energy, Office of Science, Office of Basic Energy Sciences, Materials Sciences Engineering Division. Use of the Advanced Photon Source was supported by the U.S. Department of Energy, Office of Science, Office of Basic Energy Sciences, under Contract No. DE-AC0206CH11357. The work at DESY, x-ray measurements, sample preparation, and marker-based nanotransfer, was supported by the EU-H2020 research and innovation program under Grant Agreement No. 654360 NFFAEurope (T. F. K., H. R., C. S., A. S.) and the Helmholtz Associations Initiative and Networking Fund and the Russian Science Foundation, Grant No. HRSF-0002 (L. G., Y. Y. K., I. A. V.). The use of the FIB dual beam instrument granted by BMBF (5K13WC3, PT-DESY) is acknowledged. We thank S. Kulkarni and A. Jeromin (DESY NanoLab [22]) for marking the regions of interest by IBID/EBID and the SEM postanalysis. One of the authors (T. K.) thanks the Japanese Society for the Promotion of Science (JSPS) for JSPS Postdoctoral Fellowships for Research Abroad.

*hyou@anl.gov

†Present address: Tesla, Inc., 3500 Deer Creek Road, Palo Alto, California 94304, USA

[1] G. A. Somorjai and Y. Li, Introduction to Surface Chemistry and Catalysis, 2nd ed. (Wiley, Hoboken, NJ, 2010).

[2] G. Ertl, Reactions at Solid Surfaces (Wiley, New York, 2009).

[3] C. Burda, X. B. Chen, R. Narayanan, and M. A. El-Sayed, Chem. Rev. 105, 1025 (2005).

[4] V. Komanicky, H. Iddir, K.-C. Chang, A. Menzel, G. Karapetrov, D. Hennessy, P. Zapol, and H. You, J. Am. Chem. Soc. 131, 5732 (2009).

[5] F. Tao, M. E. Grass, Y. Zhang, D. R. Butcher, J. R. Renzas, Z. Liu, J. Y. Chung, B. S. Mun, M. Salmeron, and G. A. Somorjai, Science 322, 932 (2008).

[6] P. Müller, U. Hejral, U. Rütt, and A. Stierle, Phys. Chem. Chem. Phys. 16, 13866 (2014).
[7] S. B. Maisel, T. C. Kerscher, and S. Müller, Acta Mater. 60, 1093 (2012).

[8] C. Steiner, B. Schönfeld, M. J. Portmann, M. Kompatscher, G. Kostorz, A. Mazuelas, T. Metzger, J. Kohlbrecher, and B. Demé, Phys. Rev. B 71, 104204 (2005).

[9] M. A. Pfeifer, G. J. Williams, I. A. Vartanyants, R. Harder, and I. K. Robinson, Nature (London) 442, 63 (2006).

[10] I. Robinson and R. Harder, Nat. Mater. 8, 291 (2009).

[11] M. Abuin, Y. Y. Kim, H. Runge, S. Kulkarni, S. Maier, D. Dzhigaev, S. Lazarev, L. Gelisio, C. Seitz, M.-I. Richard, T. Zhou, V. Vonk, T.F. Keller, I. A. Vartanyants, and A. Stierle,, ACS Appl. Nano Mater. 2, 4818 (2019).

[12] A. Ulvestad, J. N. Clark, R. Harder, I. K. Robinson, and O. G. Shpyrko, Nano Lett. 15, 4066 (2015).

[13] X. P. Liu, M. A. G. Aranda, B. Chen, P. Wang, R. Harder, and I. Robinson, Crystal Growth Des. 15, 3087 (2015).

[14] W. Cha, S. Song, N. C. Jeong, R. Harder, K. B. Yoon, I. K. Robinson, and H. Kim, New J. Phys. 12, 035022 (2010).

[15] W. Cha et al., Nat. Mater. 12, 729 (2013).

[16] I. A. Vartanyants and I. K. Robinson, J. Phys. Condens. Matter 13, 10593 (2001).

[17] R. Harder, M. A. Pfeifer, G. J. Williams, I. A. Vartaniants, and I. K. Robinson, Phys. Rev. B 76, 115425 (2007).

[18] W. Hu, X. J. Huang, and H. F. Yan, J. Appl. Crystallogr. 51, 167 (2018).

[19] A. G. Shabalin, O. M. Yefanov, V. L. Nosik, V. A. Bushuev, and I. A. Vartanyants, Phys. Rev. B 96, 064111 (2017).

[20] W. Cha, Y. Liu, H. You, G. B. Stephenson, and A. Ulvestad, Adv. Funct. Mater. 27, 1700331 (2017).

[21] See Supplemental Material at http://link.aps.org/ supplemental/10.1103/PhysRevLett.123.246001 for experimental and data analysis details, which includes Refs. [22-26].

[22] A. Stierle, T. F. Keller, H. Noei, V. Vonk, and R. Roehlsberger, J. Large-Scale Res. Facil. 2, A76 (2016).

[23] H. N. Chapman et al., J. Opt. Soc. Am. A 23, 1179 (2006).

[24] T. Kimura et al., Nat. Commun. 5, 3052 (2014).

[25] A. Ulvestad, A. Singer, J. N. Clark, H. M. Cho, J. W. Kim, R. Harder, J. Maser, Y. S. Meng, and O. G. Shpyrko, Science 348, 1344 (2015).

[26] D. Shapiro et al., Proc. Natl. Acad. Sci. U.S.A. 102, 15343 (2005).

[27] T. Kawaguchi, W. Cha, V. Latyshev, S. Vorobiov, V. Komanicky, and H. You, J. Korean Phys. Soc. 75, 528 (2019).

[28] W. Yang, X. Huang, R. Harder, J. N. Clark, I. K. Robinson, and H.-k. Mao, Nat. Commun. 4, 1680 (2013).

[29] J. N. Clark, X. Huang, R. Harder, and I. K. Robinson, Nat. Commun. 3, 993 (2012).

[30] C.-c. Chen, J. Miao, C. W. Wang, and T. K. Lee, Phys. Rev. B 76, 064113 (2007).

[31] V. Komanicky, D. C. Hennessy, H. Iddir, P. Zapol, and H. You, Electrochim. Acta 109, 440 (2013).

[32] W. M. Yim and R. J. Paff, J. Appl. Phys. 45, 1456 (1974).

[33] J. W. Arblaster, Platinum Met. Rev. 41, 184 (1997).

[34] J. W. Arblaster, Platinum Met. Rev. 57, 127 (2013).

[35] T. Li, P. A. J. Bagot, E. A. Marquis, S. C. E. Tsang, and G. D. W. Smith, Catalysis Today 175, 552 (2011).

[36] P. Shewmon, Diffusion in Solids, 2nd ed. (Wiley, New York, 1989). 
[37] O. Muller and R. Rustum, J. Less-Common Met. 16, 129 (1968).

[38] U. Hejral, D. Franz, S. Volkov, S. Francoual, J. Strempfer, and A. Stierle, Phys. Rev. Lett. 120, 126101 (2018).
[39] T. B. Reed, Free Energy of Formation of Binary Compounds (MIT Press, Cambridge, MA, 1971).

[40] Z. Wang, J. Ansermet, C. P. Slichter, and J. H. Sinfelt, J. Chem. Soc., Faraday Trans. 84, 3785 (1988). 